\title{
Modeling interactions between Human Equilibrative Nucleoside Transporter-1 and other factors involved in the response to gemcitabine treatment to predict clinical outcomes in pancreatic ductal adenocarcinoma patients
}

\author{
Francesca Tavano ${ }^{1}$, Andrea Fontana ${ }^{2}$, Fabio Pellegrini ${ }^{2,3}$, Francesca Paola Burbaci ${ }^{1}$, Francesca Rappa ${ }^{4,5}$, \\ Francesco Cappello, ${ }^{4,5}$, Massimiliano Copetti ${ }^{2}$, Evaristo Maiello ${ }^{6}$, Lucia Lombardi ${ }^{6}$, Paolo Graziano ${ }^{7}$, \\ Manlio Vinciguerra ${ }^{1,4,8}$, Fabio Francesco di Mola ${ }^{9}$, Pierluigi di Sebastiano ${ }^{9}$, Angelo Andriulli ${ }^{1}$ and Valerio Pazienza ${ }^{1 *}$
}

\begin{abstract}
Background: Pancreatic ductal adenocarcinoma (PDAC) is an extremely aggressive malignancy, characterized by largely unsatisfactory responses to the currently available therapeutic strategies. In this study we evaluated the expression of genes involved in gemcitabine uptake in a selected cohort of patients with PDAC, with well-defined clinical-pathological features.

Methods: mRNA levels of hENT1, CHOP, MRP1 and DCK were evaluated by means of qRT-PCR in matched pairs of tumor and adjacent normal tissue samples collected from PDAC patients treated with gemcitabine after surgical tumor resection. To detect possible interaction between gene expression levels and to identify subgroups of patients at different mortality/progression risk, the RECursive Partitioning and Amalgamation (RECPAM) method was used.

Results: RECPAM analysis showed that DCK and CHOP were most relevant variables for the identification of patients with different mortality risk, while hENT1 and CHOP were able to identify subgroups of patients with different disease progression risk. Conclusion: hENT1, CHOP, MRP1 and DCK appear correlated to PDAC, and this interaction might influence disease behavior.
\end{abstract}

Keywords: Pancreatic ductal adenocarcinoma, hENT1, CHOP, MRP1, DCK, RECPAM

\section{Introduction}

Pancreatic ductal adenocarcinoma (PDAC) is the fourth leading cause of cancer-related death $[1,2]$ with a poor prognosis due to the early undetectable symptoms and the lack of effective treatment which are responsible for the high lethality [3,4]. There is an urgent need for additional biomarkers which can predict the PDAC onset, or for implementing new effective therapeutic strategies.

\footnotetext{
* Correspondence: pazienza_valerio@yahoo.it

'Gastroenterology Unit, I.R.C.C.S. "Casa Sollievo della Sofferenza" Hospital San Giovanni Rotondo (FG) Italy, viale dei Cappuccini n.1, San Giovanni Rotondo, FG 71013, Italy

Full list of author information is available at the end of the article
}

Gemcitabine is considered the standard of care for the treatment of patients with locally advanced and metastatic PDAC, with either curative or palliative intent $[5,6]$. Gemcitabine was synthesized in early 1980s and chemically is a nucleoside analogue (similar to cytosine) which displays two fluorines on the carbon 2', instead of the hydrogen atoms, conferring it tumor growth arrest properties.

Recently, research efforts were boosted in order to find biomarkers effective into predict the clinical benefits of gemcitabine in PDAC $[7,8]$. Among the factors involved in the gemcitabine response pathway, human equilibrative nucleoside transporter 1 ( $h E N T 1)$ has been reported

\section{Ciomed Central}

(c) 2014 Tavano et al.; licensee BioMed Central Ltd. This is an Open Access article distributed under the terms of the Creative Commons Attribution License (http://creativecommons.org/licenses/by/4.0), which permits unrestricted use, distribution, and reproduction in any medium, provided the original work is properly credited. The Creative Commons Public Domain Dedication waiver (http://creativecommons.org/publicdomain/zero/1.0/) applies to the data made available in this article, unless otherwise stated. 
as the main mediator of gemcitabine uptake across plasma membranes [9], and it has been associated to gemcitabine-dependent effects in several in vitro and in vivo studies [10-14]. The deoxycytidine kinase (DCK), represents another key enzyme involved in gemcitabine phosphorylation/activation, and it has also been implicated in gemcitabine resistance [15], as well as the multidrug resistance-associated protein 1 (MRP1) which is involved in chemotherapy resistance in human pancreatic cancer [16,17]. Further, CCAAT-enhancer-binding protein homologous protein $(C H O P)$ represents a stressinduced transcription factor involved not only in the cell cycle and apoptosis [18], but also in the transcriptional down-regulation of $h E N T 1$ expression [19].

Herein we analyzed potential changes in the expression levels of $h E N T 1, D C K, M R P 1$ and $C H O P$, as are the main factors involved in the gemcitabine response pathway and they are involved in gemcitabine uptake and activation, in biopsies of PDAC patients in order to find possible associations between the mutual expression levels of these genes and clinical pathological features in a selected and well characterized cohort of patients with PDAC.

\section{Material and methods Clinical samples}

The training cohort included tissues specimens collected from 26 patients with pancreatic ductal adenocarcinoma (PDAC). These patients were selected among a total of 76 subjects, with final pathological diagnosis of PDAC, who underwent pancreatic resection and at our hospital IRCCS "Casa Sollievo della Sofferenza" San Giovanni Rotondo (Italy), between March 2008 and May 2014. The inclusion criteria included: availability of matched pairs of tumor and adjacent normal tissue sample (54 out of 76), adjuvant chemotherapy with gemcitabine administered after complete resection of pancreatic cancer in absence of neo-adiuvant treatments (26 out of 54), and complete follow-up data, including either clinical examination of CA 19-9 serum marker and monitor of response to treatment at regular intervals (26 out of 26). In details, all the 26 patients fulfilling these criteria, weekly received gemcitabine at a dose of $1,000 \mathrm{mg} / \mathrm{m} 2$ for 7 weeks as an induction phase. After this phase, CA 19-9 levels were elevated in 10 patients. This patients underwent to computed tomography scan that showed visceral metastases (liver, mesenteric, lung etc.), therefore were evaluated for a first line treatment. The remaining patients with normal CA 19-9 levels, entered the chemoradiotherapy phase of the treatment. In five patients, gemcitabine $400 \mathrm{mg} / \mathrm{m} 2$ weekly $\times 3$ every 28 days for 2 cycles, and concurrent radiotherapy, for a total dose of 50.4 Gy in 28 fractions were prescribed. In one patient, at the end of radiotherapy, gemcitabine was continued as maintenance.
The others 8 patients received only gemcitabine at the same doses above-mentioned without radiotherapy. The study was approved by the hospital ethical committee. Tissue specimens were immediately frozen in liquid nitrogen, and stored at $-80^{\circ} \mathrm{C}$ until RNA extraction. All the patients signed the informed consent before tissues collection. Demographics and clinical-pathological characteristics of patients are listed in Table 1.

\section{RNA isolation}

Cryostat representative sections of the tumor were morphologically evaluated and neoplastic cellularity was enriched by microdissection of the most cellular areas. Total RNA was extracted from fresh frozen specimens by means of TRIzol $^{\odot}$ Reagent (Invitrogen, Milano, Italy) and subsequently purified using RNeasy ${ }^{\circ}$ Mini Kit and digestion with DNase I. (Qiagen, Milano, Italy), according to manufacturer's recommendations. RNA concentration and purity (A260:A280 > 2.0; A260/A230 > 1.8) were controlled by NanoDrop Spectrophotometer (Thermo Fisher, Waltham, MA, USA).

\section{Quantitative Real Time PCR}

Expression analyses were performed using QuantiFast Sybr Green PCR kit (Qiagen, Milano, Italy), following the one-step protocol: cDNA was first synthesized from 60 ng total RNA, and then amplified by means of the Sybr Green QuantiTect Primer (Qiagen, Milano, Italy): hENT1 (QT010000083), CHOP (QT00082278), MRP1 (QT00061159) and DKC (QT00000392). Reactions were set up in 96-well plates and loaded onto 7700 Real-Time PCR System (Applied Biosystems, Foster City, CA). Optical data obtained were analyzed using the SDS software package (version 1.9.1; Applied Biosystems, Foster City, CA). Expression levels of target gene were obtained using the comparative method of relative quantification, after normalization for the housekeeping control gene Glyceraldehyde-3-phosphate dehydrogenase GAPDH (Sigma Aldrich, Milano, Italy), as previously performed [20].

\section{Immunohistochemistry}

Formalin-fixed, paraffin-embedded PDAC sections were immunostained as already described [21] by using iVIEW DAB Detection Kit for Ventana BenchMark XT automated slide stainer on human biopsies. Primary antibodies for hENT1 was purchased from Santacruz (cat. n. sc-134501) and diluted 1:100. Appropriate positive controls, as well as non-immune serum for negative controls, were run concurrently.

Normal pancreatic tissue samples were obtained from OriGene (Rockville, MD, USA) http://www.origene.com/ tissue/tissue_qc.aspx. hENT1 immunoreactivity was evaluated in blind by two expert pathologists (FR and FC) 
Table 1 Clinical and pathological features of 26 patients with Pancreatic Ductal Adenocarcinoma treated with Gemcitabine after pancreatic resection

\begin{tabular}{|c|c|}
\hline Age at diagnosis (years), median (Q1-Q3) & $64.5(51-73)$ \\
\hline Gender, male/female (\%male) & $17 / 9(65)$ \\
\hline \multicolumn{2}{|l|}{ Smoking habit, n (\%) } \\
\hline No & $10(48)$ \\
\hline Yes & $8(38)$ \\
\hline Ex & $3(14)$ \\
\hline Missing information & 5 \\
\hline \multicolumn{2}{|l|}{ Alcohol Use, n (\%) } \\
\hline No & $18(95)$ \\
\hline Yes & $1(5)$ \\
\hline Missing information & 7 \\
\hline Jaundice, y/n (\%y) & $15 / 11(58)$ \\
\hline Diabetes mellitus, y/n (\%y) & 9/17 (35) \\
\hline Familial*, y/n (\%y) & $5 / 21(19)$ \\
\hline Previous Neoplasia, y/n (\%y) & $2 / 24(8)$ \\
\hline $\begin{array}{l}\text { Preoperative serum CAE levels ( } \mathrm{ng} / \mathrm{ml}) \text {, median (Q1- } \\
\text { Q3) }\end{array}$ & $3.2(2.0-6.0)$ \\
\hline $\begin{array}{l}\text { Preoperative serum CA 19-9 levels }(\mathrm{U} / \mathrm{ml}) \text {, median } \\
\text { (Q1-Q3) }\end{array}$ & $\begin{array}{l}200.4(53.5- \\
335.9)\end{array}$ \\
\hline Size (cm), median (Q1-Q3) & $3(2.4-3.5)$ \\
\hline \multicolumn{2}{|l|}{ Tumour type, n (\%) } \\
\hline Adenocarcinoma & $22(85)$ \\
\hline Adenocarcinoma Mucinous & $4(15)$ \\
\hline \multicolumn{2}{|l|}{ Tumour grading, n (\%) } \\
\hline G1: well differentiated & $5(20)$ \\
\hline G2: moderately differentiated & $10(40)$ \\
\hline G3: poorly differentiated & $10(40)$ \\
\hline Missing information & 1 \\
\hline \multicolumn{2}{|l|}{ T: Tumour size, n (\%) } \\
\hline $\mathrm{T} 1$ & $1(4)$ \\
\hline $\mathrm{T} 2$ & $2(8)$ \\
\hline T3 & $23(88)$ \\
\hline \multicolumn{2}{|l|}{ N: regional lymph nodes, n (\%) } \\
\hline No & $3(12)$ \\
\hline N1 & $23(88)$ \\
\hline Lymph nodes ratio, median (Q1-Q3) & $0.24(0.07-0.47)$ \\
\hline \multicolumn{2}{|l|}{ Tumour stage, n (\%) } \\
\hline$\| A$ & $3(12)$ \\
\hline$\| \mathrm{B}$ & $23(88)$ \\
\hline Perineural Invasion, y/n (\% y) & $14 / 12(54)$ \\
\hline Vascular Invasion, y/n (\% y) & $3 / 23(12)$ \\
\hline \multicolumn{2}{|l|}{ Margins of resection, n (\%) } \\
\hline R0: negative resection margins & $18(69)$ \\
\hline R1: microscopic positive resection margins & $8(31)$ \\
\hline
\end{tabular}

Table 1 Clinical and pathological features of 26 patients with Pancreatic Ductal Adenocarcinoma treated with Gemcitabine after pancreatic resection (Continued)

\begin{tabular}{ll}
\hline $\begin{array}{l}\text { Postoperative serum CAE levels (ng/ml), median (Q1- } \\
\text { Q3) }\end{array}$ & $3.4(2.0-4.5)$ \\
$\begin{array}{l}\text { Postoperative serum CA 19-9 levels (U/ml), median } \\
\text { (Q1-Q3) }\end{array}$ & $10.4(7.3-36.2)$ \\
Treatment with Gemcitabine & \\
$\quad$ Nr. Cycles, median (Q1-Q3) & $5(3-7)$ \\
$\quad \mathrm{Nr}$. Cycles < 6, n (\%) & $10(39)$ \\
Nr. Cycles $>6, \mathrm{n}(\%)$ & $16(62)$ \\
Overall Follow-up (yrs), median (Q1-Q3) & $1.4(0.9-2.3)$ \\
Disease progression Follow-up (yrs), median (Q1-Q3) & $0.9(0.6-1.3)$ \\
Mortality rate & $16 / 45(35)$ \\
Disease Progression rate** & $17 / 29(58)$
\end{tabular}

*For neoplasia or chronic pancreatitis; ${ }^{* *}$ Number of events/person-years (expected number of events per 100 person-years).

assessing a semiquantitative scoring system in ten high power fields (10HPF, $\mathrm{X} 400)$ according to a semiquantitative scale (-: 0\%; +: 1-33\%; ++: 34-66\%; +++: 67-100\%).

\section{Statistical methods}

Baseline patients' characteristics were reported as frequency (percentages) and mean \pm standard deviation (SD), along with median and lower (Q1) and upper (Q3) quartiles range, for categorical variables and continuous variables, respectively. Normal distribution assumption was checked by means of Q-Q plot, Shapiro-Wilks and Kolmogorov-Smirnov tests. To assess the presence of down/over regulation of genes expression in tumors compared to normal samples, one-sample $t$-test was performed using logarithm-transformed gene expression values. Correlations between (log-transformed) gene expression levels were assessed estimating Pearson's correlation coefficients $(\mathrm{r})$, whereas comparisons between log-transformed gene expression levels and categorical clinical variables were assessed using two-sample $t$-test or ANOVA models, respectively. Time to disease progression was defined as the time between the date of the surgery (baseline) and the date of the first progression event. Time to death was defined as the time between the baseline and the date of death. For subjects who did not experience any event, time variable was defined as the time between the baseline and the date of the last available clinical follow-up.

Incidence rates for events (i.e. disease progression or death, separately) were calculated as the number of events divided by the estimated persons-years, and eventually multiplied by 100 .

To evaluate interactions between $h E N T 1, C H O P, D C K$, MRP1 genes only, and between hENT1, CHOP, DCK, $M R P 1$ genes along with all patients' clinical variables, 
identifying distinct and homogeneous subgroups of patients in terms of progression-free survival (PFS) and overall survival (OS), the RECursive Partitioning and AMalgamation (RECPAM) method was used [22,23]. The tree-growing algorithm estimates hazard ratios (HR), along with 95\% confidence interval (95\% CI), from a Cox proportional hazards regression model using appropriate covariates, as candidate splitting variables.

At each partitioning step, the method chooses the covariate and its best binary split to maximize the difference in the outcome of interest (i.e. PFS or OS). The algorithm stops when user defined conditions (stopping rules) are met (i.e. at least one event and at least three subjects per leaf). To obtain more robust and stable split (cut-off), a permutation approach was adopted to choose the best splitting variable. Furthermore, survival curves were drawn, for each final RECPAM class, from Cox proportional hazard models.

Moreover, all clinical features which could affect PDAC risk and clinical outcome of patients (i.e. diabetes mellitus, family history for neoplasia, tumor stage, positive surgical margins of resection, presence of vascular invasion, number of cycles of treatment with gemcitabine.) were compared between final RECPAM classes, using the Kruskal-Wallis and Fisher exact tests (due to non-normal data distribution and small sample size) for continuous and categorical variables, respectively.

A p value $<0.05$ was considered for statistical significance. All analyses were performed using SAS Release 9.3 (SAS Institute, Cary, NC, USA). For the RECPAM analysis a SAS macro routine, written by one of the authors (F. Pellegrini), was used.

\section{Results}

\section{Relative expression levels of hENT1, CHOP, MRP1 and DCK in PDAC biopsies}

Looking at median of gene expression levels in tumors compared to adjacent normal tissues, hENT1 downregulation in tumor samples was found (fold-change = 0.53, Q1-Q3 = 0.23-0.94, $\mathrm{p}=0.006$ ), (Figure 1). No differences in CHOP, MRP1 and DCK expression levels were observed in tumors compared to normal tissues (CHOP: fold-change $=1.02, \quad \mathrm{Q} 1-\mathrm{Q} 3=0.16-2.02, \quad \mathrm{p}=0.266 ; \quad M R P 1$ : fold-change $=0.58, \quad \mathrm{Q} 1-\mathrm{Q} 3=0.22-1.21, \quad \mathrm{p}=0.203 ; \quad D C K$ fold-change $=1.15, \mathrm{Q} 1-\mathrm{Q} 3=0.28-2.36, \mathrm{p}=0.370$ ) Figure 1. Furthermore, MRP1 expression levels were significantly correlated with those of both $h E N T 1(\mathrm{r}=0.53, \mathrm{p}=0.006)$ and CHOP $(\mathrm{r}=0.42, \mathrm{p}=0.032)$.

\section{Associations with clinical phenotypes}

To establish whether mRNA levels were associated with specific diseases phenotypes, we analyzed the possible associations of genes expression with age at diagnosis, gender, smoking habit, alcohol use, presence of jaundice,

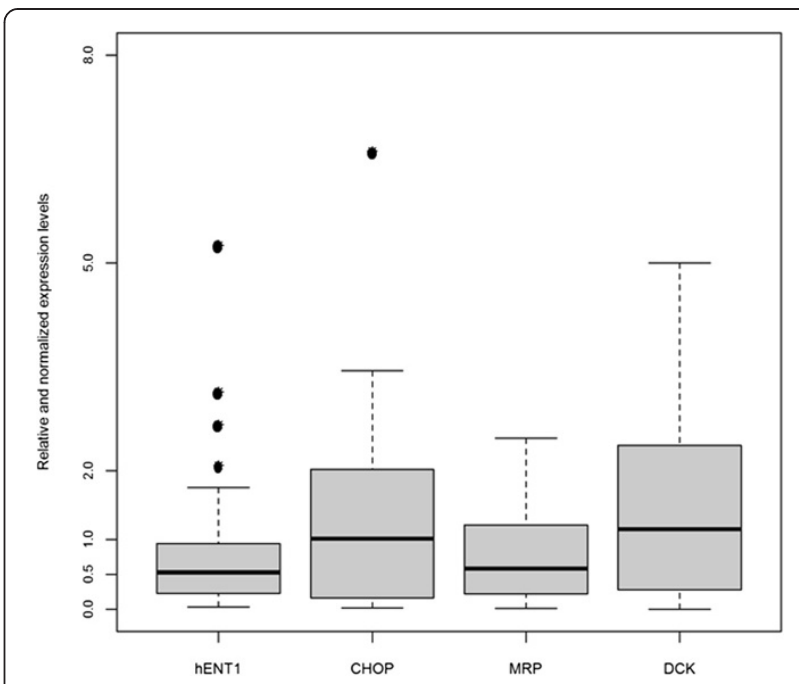

Figure 1 Boxplot of relative expression levels of $h E N T 1, C H O P$, MRP1 and DCK in matched pairs of tumor and normal samples from patients with Pancreatic Ductal Adenocarcinoma. Each box highlights median (horizontal black bar), interquartile range (Q1-Q3) and lower and upper adjacent values (vertical bars) for each gene. Genes expression values are reported in log scale (y-axis). ${ }^{*} p<0.001$.

diabete mellitus, family history for neoplasia, serum levels of carcinoembryonic antigen (CEA), tumor markers, tumor grading, tumor size, tumor histological type, lymph node spreading, staging of tumor, resection margins and medical therapy. In details, levels of hENT1 were associated with jaundice and the resection margins of patients at diagnosis. hENT1 resulted differentially expressed in jaundiced patients compared to those without jaundice (jaundiced: fold-change $=0.61, \mathrm{Q} 1-\mathrm{Q} 3=$ 0.32-1.76 vs not jaundiced: fold-change $=0.25, \mathrm{Q} 1-\mathrm{Q} 3=$ $0.11-0.84 ; \mathrm{p}=0.047$ ), and was differentially expressed in patients with evidence of tumor infiltration of the resection margins (R1) with respect to those with resection margins free from tumor cells (R0), (R1: fold-change = 1.35, Q1-Q3 = 0.32-2.37 vs R0: fold-change $=0.51$, Q1$\mathrm{Q} 3=0.21-0.77 ; \mathrm{p}=0.030)$ showing a trend without reaching a statistical significance for the age (hENT1: $\mathrm{r}=0.36, \mathrm{p}=0.067)$. CHOP resulted down-regulated in patients with family history of cancer (F1: fold-change = 0.11, Q1-Q3 = 0.09-0.16 vs F0: fold-change = 1.23, Q1$\mathrm{Q} 3=0.24-2.39 ; \mathrm{p}=0.044)$ and over-expressed in patients who had a previous neoplasia (PN1: fold-change $=31.77$, $\mathrm{Q} 1-\mathrm{Q} 3=1.57-61.98$ vs PN0: fold-change $=0.66$, Q1-Q3 = $0.13-1.93 ; \mathrm{p}=0.033)$. Interestingly, expression levels of $D C K$ were found down regulated in mucinous adenocarcinoma (AM: fold change $=0.36, \mathrm{Q} 1-\mathrm{Q} 3=0.14-0.57$ vs $\mathrm{A}$ : fold change $=1.34 \mathrm{Q} 1-\mathrm{Q} 3=0.34-2.94 \quad \mathrm{p}=0.016$ ). Finally, MRP1 expression did not show any statically significant association with the clinical phenotypes taken in consideration. 


\section{Survival analysis}

In the overall sample, $16 / 26(61.5 \%)$ patients died during the follow-up and $17 / 26(65.3 \%)$ had a disease progression. Specifically, the overall mortality rate was 35 events per 100 person/years (median follow-up of 1.4 years), whereas the overall disease progression rate was 58 events per 100 person/years (median follow-up of 0.9 years).

Focusing on the investigation of interactions between hENT1, CHOP, MRP1 and DCK genes on OS and PFS outcome, RECPAM tree-growing algorithm identified three classes at different mortality risks. As shown in Figure 2A, the reference class (Class 3 ) is represented by the subgroup with the lowest mortality, and all the HRs are estimated with respect to this reference class. In details, patients with DCK values $\leq 0.27(n=6)$ identified the reference class $(\mathrm{HR}=1)$, whereas those with $\mathrm{DCK}>$ 0.27 and $\mathrm{CHOP} \leq 0.25(\mathrm{n}=6)$ identified the class with the highest mortality risk (Class $1, \mathrm{HR}=9.10,95 \% \mathrm{CI}=$ 1.50-55.04). Furthermore, patients with DCK $>0.27$ and CHOP $>0.25(\mathrm{n}=14)$ identified the intermediate risk class (Class $2, \mathrm{HR}=3.23,95 \% \mathrm{CI}=0.64-16.23$ ). The overall mortality rates (reported as the number of events per 100 person-years), along with median follow-up time, were: 63 (16.3 months), 35 (13.7 months), 23 (35.4 months) for Classes 1,2 and 3, respectively. (Figure 2A).

When looking at the clinical pathological features of patients within each RECPAM classes (please see profiles at the bottom of Figure 2A), we found that preoperative serum levels of CEA were higher in patients with lowest mortality risk (and vice versa) while, although not statistically significant, the percentage of subjects with jaundice and stage IIB-cancer patients was slight higher within intermediate and highest mortality risk classes. Interestingly, half of the patients with highest mortality risk had family history of cancer and were affected from diabetes mellitus, whereas about twothirds of them were treated with gemcitabine for less than six cycles. On the other hand, a significantly higher percentage of patients with positive surgical margins were observed within intermediate risk class, as compared to the other classes $(\mathrm{p}=0.007)$.

Moreover, RECPAM algorithm also identified three classes at different disease-progression risk: as shown in Figure $2 \mathrm{~B}$, patients with $h E N T 1 \leq 0.11(\mathrm{n}=3)$ identified the reference class $(\mathrm{HR}=1)$, whereas those with $h E N T 1>0.11$ and $D C K \leq 1.43(\mathrm{n}=14)$ identified the class with the highest disease-progression risk (Class $1, \mathrm{HR}=5.74$, 95\% CI $=1.01-32.43)$. Furthermore, patients with $h E N T 1>0.11$ and $D C K>1.43(\mathrm{n}=9)$ identified the intermediate risk class (Class $2, \mathrm{HR}=1.63,95 \% \mathrm{CI}=0.31$ 8.57). Disease progression rates (reported as the number of events per 100 person-years), along with median follow-up time, were: 90 (7.7 months), 48 (11.2 months),
26 (23.0 months) for Classes 1,2 and 3, respectively. Figures 2A-B reports survival curves, identified by RECPAM analyses for OS and PFS, respectively.

When looking at the clinical pathological features of patients within each RECPAM classes (please see profiles at the bottom of Figure 2B), we found that patients with positive surgical margins belonged to Class 1 and 2, although no statistically significance was observed.

When clinical variables were also included as candidate splitting variables, along with $h E N T 1, C H O P, D C K$, $M R P 1$ genes, RECPAM tree-growing algorithm identified three classes at different mortality risks: as shown in Figure 3A, patients with $D C K \leq 0.27(n=6)$ identified the reference class $(\mathrm{HR}=1)$, whereas those with $D C K>0.27$ and received $<6$ cycles of gemcitabine $(n=9)$ identified the class with the highest mortality risk (Class $1, \mathrm{HR}=$ 21.41, 95\% CI $=2.48-185.08)$. Furthermore, patients with $D C K>0.27$ and received $>6$ cycles of gemcitabine $(n=11)$ identified the intermediate risk class (Class $2, \mathrm{HR}=3.38$, 95\% CI = 0.70-16.41).

Similarly, RECPAM tree-growing algorithm identified three classes at different disease-progression risks: as shown in Figure 3B, patients with lymph nodes ratio (LNM, i.e. the total involved lymph nodes among the total number of resected lymph nodes) $\leq 0.33(\mathrm{n}=15)$ identified the reference class $(\mathrm{HR}=1)$, whereas those with LNM $>0.33$ and MRP1 levels $\leq 1.21(n=6)$ identified the class with the highest disease-progression risk (Class 1, $\mathrm{HR}=21.20,95 \% \mathrm{CI}=3.55-126.75$ ). Furthermore, patients with $\mathrm{LMN}>0.33$ and MRP1 levels $>1.21$ $(\mathrm{n}=5)$ identified the intermediate risk class (Class $2, \mathrm{HR}=$ $1.46,95 \% \mathrm{CI}=0.36-5.95)$.

\section{Immunohistochemistry}

In order to perform a pilot evaluation of hENT1 protein localization/expression, immunohistochemical evaluations were carried out on 13 out of the 26 PDAC specimens used in the previous transcription analysis. Figure 4A and B shows 7 out of 13 selected representative immunoistochemistry of cases with highest hENT1 mRNA (1 V-15660), intermediate (1 M-17396; 193551-10 and I2012-016339) or lowest levels (1 T17785; 1 M17536 and I2011-010518) among all PDAC samples. Pathological features of these samples were listed in Table 2. hENT1 was mainly localized in the cytoplasm of the tumor cells. In particular, the strongest epithelial cell immunopositivity was observed in samples displaying the lowest hENT1 mRNA expression. By contrast, samples with an intermediate $h E N T 1$ mRNA expression showed a lower positivity for hENT1 protein that was present in approximately a half of the cancer cells. Finally the only sample with the highest hENT1 mRNA expression (1V-15660) was negative for hENT1 protein immunopositivity (Figure 4A-B). 


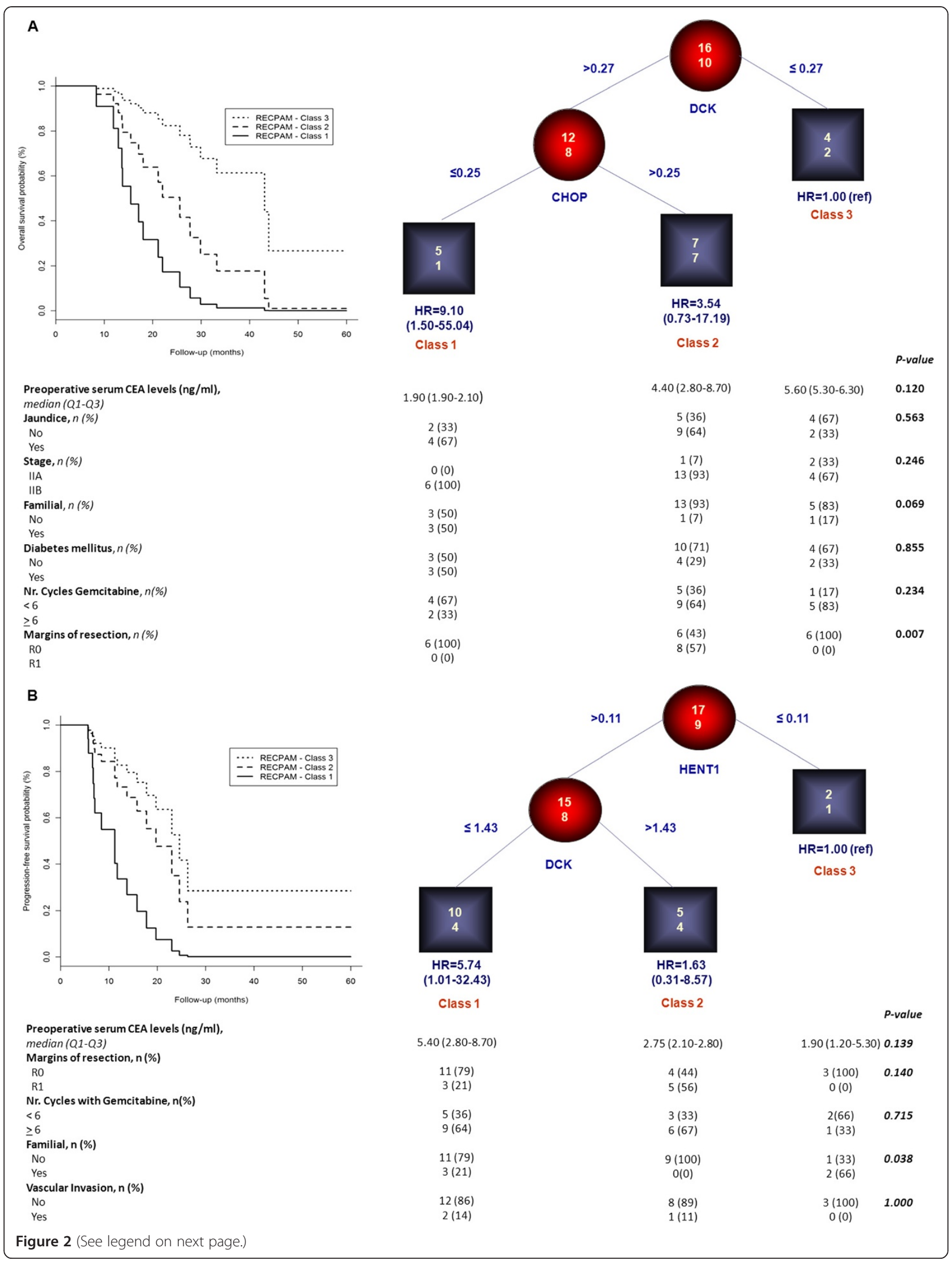


(See figure on previous page.)

Figure 2 Identification of subgroups at different risks based on gene-expression interactions: results of RECPAM analysis. A: Classes of patients with different mortality risks; B: Classes of patients with different disease progression risks. Chosen splitting variables are shown between branches, while condition sending patients to left or right sibling is on relative branch. Circles indicate subgroups of patients. Squares indicate RECPAM classes. Numbers inside circles and squares represent the number of events (top) and the number of non-events (bottom), respectively. The table placed at the bottom of the figures A-B shows patients' characteristics within each RECPAM class. Plot of survival curves, estimated from Cox proportional-hazards models, with respect to each identified RECPAM class for overall survival (panel A) and progression-free survival (panel B).

A

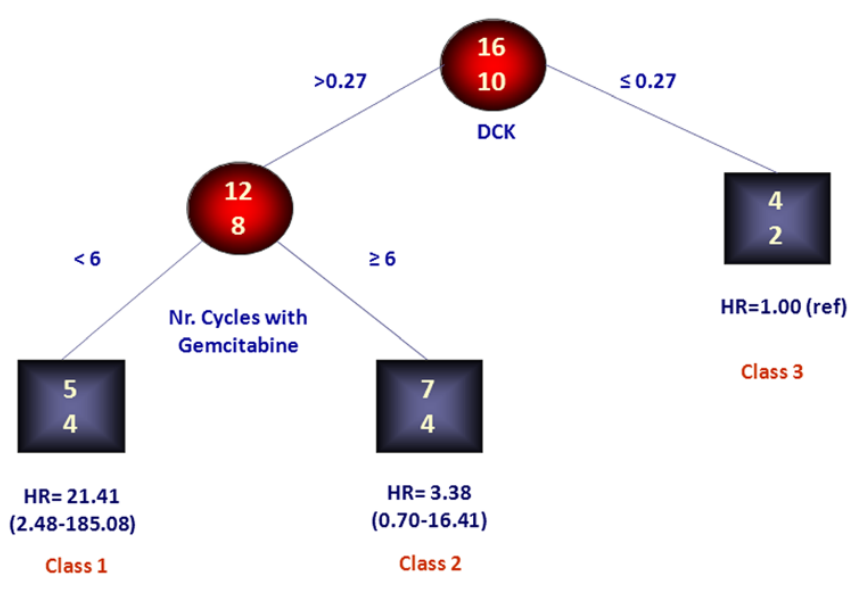

B

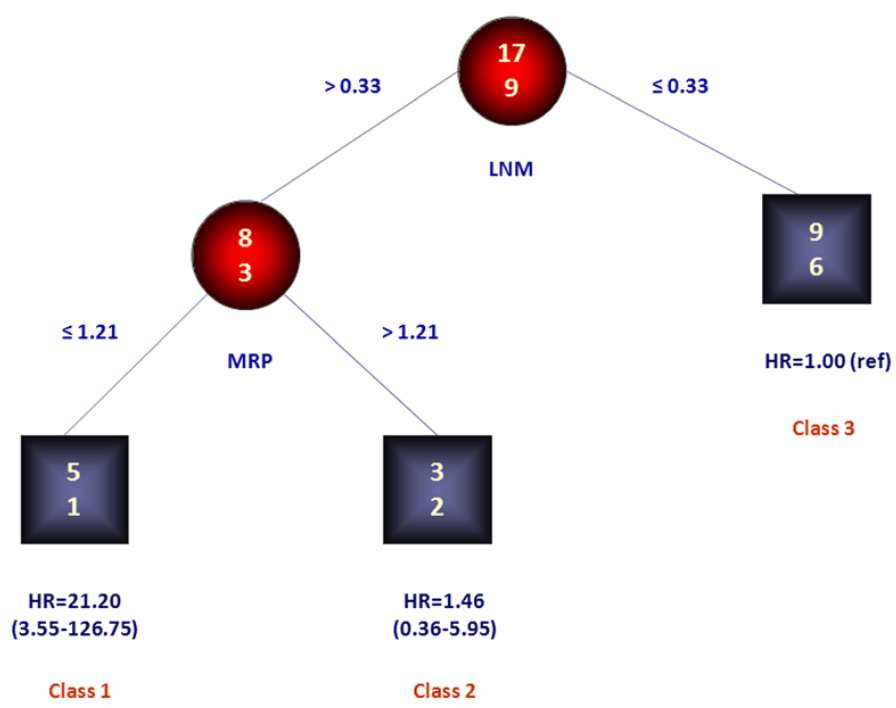

Figure 3 Identification of subgroups at different risks based on patients' clinical features-gene expression interactions: results of RECPAM analysis. A: Classes of patients with different mortality risks; B: Classes of patients with different disease progression risks. Chosen splitting variables are shown between branches, while condition sending patients to left or right sibling is on relative branch. Circles indicate subgroups of patients. Squares indicate RECPAM classes. Numbers inside circles and squares represent the number of events (top) and the number of non-events (bottom), respectively. 
Strikingly, an inverse correlation between hENT1 mRNA and protein levels was found. In fact, tissue samples from PDAC patients with higher levels of hENT1 mRNA displayed lower levels of the protein and, conversely, PDAC samples with low hENT1 protein levels showed higher levels of mRNA (Figure 4B). Noteworthy, normal pancreatic tissue displayed low hENT1 immunopositivity in the cytpoplasm and occasionally in the nuclei of the exocrine component (as indicated by the arrows in figure $4 \mathrm{C}$ ) whereas high levels of hENT1 protein were observed in either cytoplasm and nuclei of the endocrine component.

We propose the existence of a post-transcriptional feedback mechanism within PDAC cells that finely and reciprocally tune mRNA and protein levels of hENT1. In this respect, high levels of $h E N T 1$ mRNA in the most aggressive tumors could be part of a compensatory and adaptive mechanism. Mechanistic studies are warranted to support this hypothesis.

\section{Discussion}

Gemcitabine still represents the most common chemotherapeutic agent in the treatment of patients with PDAC $[5,6]$. However, nowadays cellular resistance to gemcitabine treatment represents the major problem in the clinical management of these patients [4]. Therefore, potential strategies to overcome and/or forecast chemoresistance are required in order to improve patient survival. In the present study, we examined gemcitabine related genes in a cohort of patients with PDAC underwent surgery and subsequent adjuvant treatment. To date a number of studies have evaluated hENT1 and other factors involved in gemcitabine pathway by means of functional assays in pancreatic cancer cell lines and/ or human specimens mainly by using immunoistochemistry, and transcriptional analysis in few cases [24-28]. Anyhow, our study represents the first attempt to concurrently analyze, in a well selected population of PDAC patients, mRNA expression levels of $h E N T 1$ and other genes involved in gemcitabine pathway. In the overall sample we found a down-regulation of hENT1 and $M R P 1$ in tumor compared to normal tissues, even though only hENT1 down-regulation was statistically significant. On the other hand a substantially unchanged expression of $D C K$ and $C H O P$ emerged in our samples. Data on $D C K$ were in line with those of Giovannetti and colleagues [28]. However, in this study, the authors found that $h E N T 1$ was over-expressed in tumor specimens from

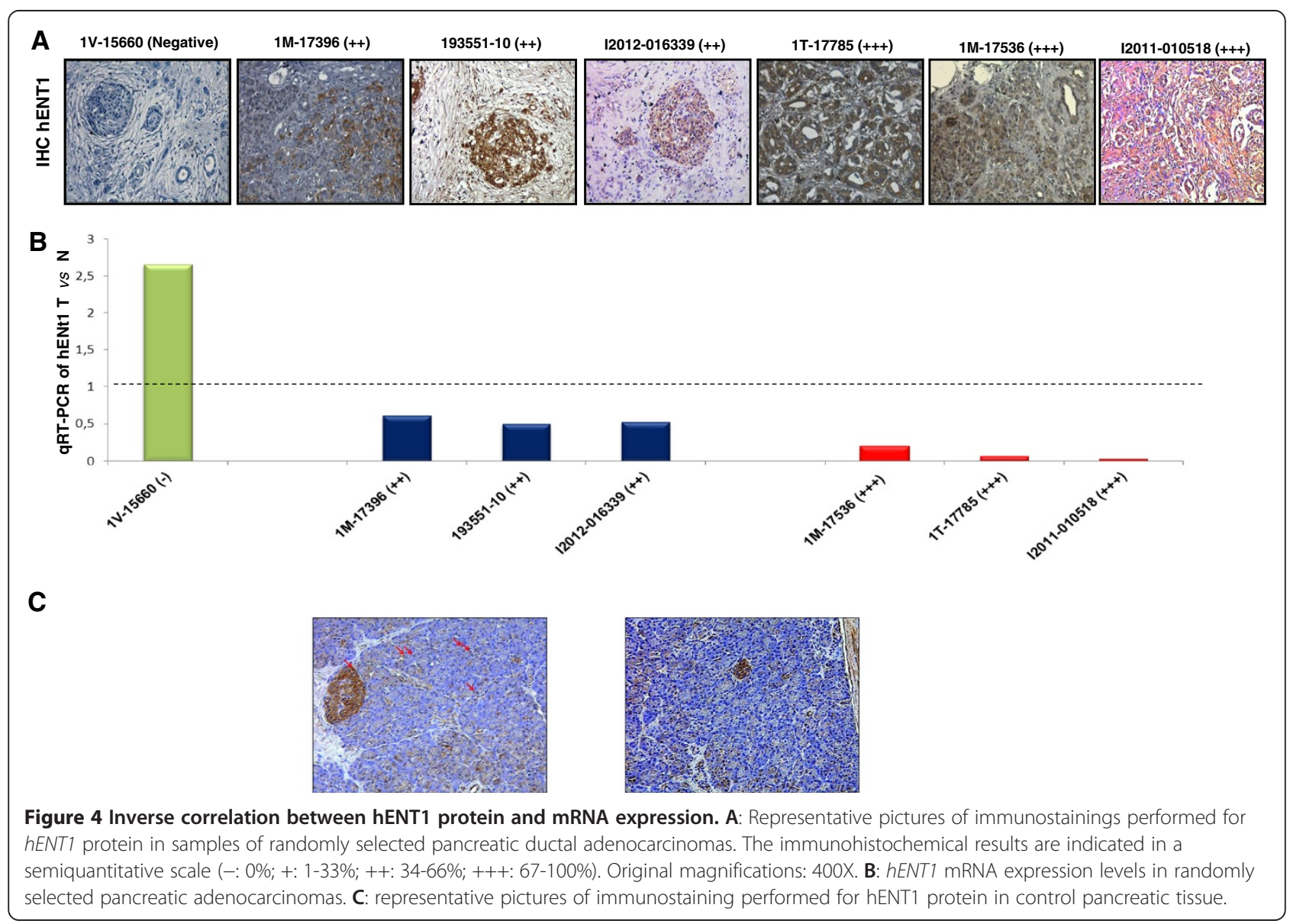


patients with pancreatic cancer. This difference could be explained by the different number of subjects considered in the two studies, as well as by the pathological characterization of clinical specimens and the surgical/ oncological treatment of patients.

In addition a direct correlation between levels of both hENT1 and CHOP with MRP1 expression emerged. These data suggest that the role of $h E N T 1$ as main mediator of gemcitabine across plasma membrane could be also affected from expression of MRP1 in PDAC tumor tissues, directly or as result of CHOP transcriptional regulation. In relation to patient clinical phenotypes the most interesting findings concern differential expression levels of $h E N T 1$ with respect to the status of resection margins, a clinical pathological feature well recognized as prognostic factor of PDAC [29,30]. On the other hand, a significant association between hENT1 and jaundice at the diagnosis was observed. Jaundice represents the most common clinical symptom, albeit belated, of the disease, and has been also recently unraveled as a risk factor for diminished survival in patients with adenocarcinoma of the head of the pancreas [29]. Jaundice represents the most common clinical symptom, albeit belated, of the disease, and has been also recently unraveled as a risk factor for diminished survival in patients with adenocarcinoma of the head of the pancreas [31]. Significant correlations were also found for $C H O P$ with respect to previous neoplasia and post-operative serum levels of CEA, one of the most common tumor markers used for clinical diagnosis of gastrointestinal and pancreatobiliary malignancies [32,33]. Finally, DCK down-regulation was associated with mucinous histotype of PDCA. Expression levels observed for the enzyme involved in gemcitabine phosphorylation/activation could explain the lower response to treatment observed in patients with mucinous disease compared to those without this pathological feature [34].

Several authors stated that higher $h E N T 1$ levels were associated with significantly longer overall survival and disease free survival in patients affected by pancreatic cancer [25-28]. As we mentioned above, the disagreement with data of Giovannetti and colleagues [28] may be due to the differences in patients enrolled in the two studies. Moreover in our cohort, $h E N T 1$ mRNA expression was inversely correlated with the protein expression levels and this also could explain the discrepancy observed in the literature among the different studies $[14,28,35]$. Our results, together with the previous observations, highlights that $h E N T 1$ protein expression would be the most appropriate predictive marker.

Nevertheless, in order to determine the usefulness of intratumoral expression of $h E N T 1$ and other factors involved in gemcitabine pathway as predictive markers of the efficacy of adjuvant gemcitabine-based chemotherapy for PDAC after operative resection, we used the RECPAM model to choose the natural cut-off points of genes expression levels for identification of patients at different-risk of mortality and disease progression. RECPAM analysis uncovered DCK and CHOP as the most important genes in distinguish patients with different mortality risk, while $h E N T 1$ and $D C K$ were able to identify subgroups of patients with different disease progression risk. Using these RECPAM models to evaluate the possible effect/contribution of patient clinical profiles on gene expression alterations, we also tested a set of variables made of the suggested risk or prognostic factors for PDAC.

\section{Conclusion}

In this study we found that intermediate mortality risk was associated with positive surgical resected margins, whereas class of patients with high mortality risk was characterized by higher percentage of patients with different PDAC risk factors (family history). In relation to disease progression we showed that family history of neoplasia was associated. Our data suggested an interaction between $D C K$ levels and the preoperative serum CEA levels and between preoperative serum CEA levels and tumor grading in relation to mortality and disease progression risk, respectively. Taken

Table 2 Pathological features of 7 patients with Pancreatic Ductal Adenocarcinoma included in the immunohistochemistry analysis

\begin{tabular}{|c|c|c|c|c|c|c|c|}
\hline & Age & Gender & Tumour type & Tumour Size $(\mathrm{cm})$ & Tumour Grading & Tumour stage & Margins of resection \\
\hline $1 \mathrm{~V}-15660$ & 78 & $m$ & PDAC & 3.05 & G2-G3 & $\| B$ & R1 \\
\hline $1 \mathrm{M}-17396$ & 69 & m & Mucinous PDAC & 3 & G1 & $\| \mathrm{B}$ & RO \\
\hline $1 \mathrm{M}-17536$ & 53 & $f$ & PDAC & 3 & G2-G3 & $\| \mathrm{A}$ & RO \\
\hline $1 \mathrm{~T}-17785$ & 65 & m & PDAC & 3 & G2-G3 & $\| B$ & RO \\
\hline 193551-10 & 73 & m & PDAC & 1,8 & G1 & $\| \mathrm{B}$ & RO \\
\hline |2011-010518 & 51 & $f$ & PDAC & 2,5 & $\mathrm{G} 2$ & $\| \mathrm{B}$ & RO \\
\hline |2012-016339 & 78 & f & PDAC & 2,4 & $\mathrm{G} 2$ & $\| \mathrm{B}$ & Ro \\
\hline
\end{tabular}

G1: well differentiated; G2: moderately differentiated; G3: poorly differentiated. R0: negative resection margins; R1:microscopic positive resection margins. Tumor stage sec. AJCC 2010. 
together all these data may help clinicians to classify disease behavior in PC patients, based on this gene expression pattern background.

In this study we found that intermediate mortality risk was associated with positive surgical resected margins, whereas class of patients with high mortality risk was characterized by higher percentage of patients with different PDAC risk factors (family history for neoplasia although not significant). In addition, classes of patients with either intermediate or high risk of PDAC showed both to be characterized by an higher trend of jaundice and by tumors stage and unsuccessful gemcitabine induction phase. Indeed, also jaundice has been recently described as important, although poorly recognized risk factor for diminished survival in patients with adenocarcinoma of the pancreas [29]. Similarly, in relation to disease progression we showed that both positive resection margins and unsuccessful gemcitabine induction phase were present within the classes of patients at higher and intermediate risk although no statistical significance was observed. Finally, our data suggested an interaction between DCK levels and the number of cycles of gemcitabine and between lymph nodes metastases and MRP1 expression in relation to mortality and disease progression risk, respectively. Taken together all these data may help clinicians to classify disease behavior in PC patients, based on this gene expression pattern background.

\section{Competing interests}

The authors declare that they have no competing interest.

\section{Authors' contributions}

VP and FT conceived the study, analyzed the data and wrote the manuscript; FPB performed qRTPCR experiments; FR, FC performed IHC experiments and FR, FC and PG evaluated the score; FFDM and PDS harvested human specimens; LL and EM assisted chemotherapy scheduling; AF, MC and FP performed the statistical analyses; PG, MV and AA critically revised the manuscript. All authors have read and approved the journal's authorship agreement. All author read and approved the final manuscript.

\section{Acknowledgment}

The study was supported by a grant from the Italian Ministry of Health through Division of Gastroenterology (RC1303GA49 to VP and RC1303GA53, RC1303GA54 to FT) IRCCS Scientific Institute and Regional General Hospital "Casa Sollievo della Sofferenza", Opera di Padre Pio da Pietrelcina, San Giovanni Rotondo (FG), Italy and by the " $5 \times 1000$ " voluntary contributions. VP and FT contributed also as PhD-student from the Department of Agricultura Science University of Foggia and Department of Biomedical Sciences, University of Chieti (Italy) respectively. We are grateful to Mimma Gioffreda for technical assistance.

\section{Author details}

${ }^{1}$ Gastroenterology Unit, I.R.C.C.S. "Casa Sollievo della Sofferenza" Hospital San Giovanni Rotondo (FG) Italy, viale dei Cappuccini n.1, San Giovanni Rotondo, FG 71013, Italy. "Unit of Biostatistics I.R.C.C.S. "Casa Sollievo della Sofferenza" Hospital San Giovanni Rotondo (FG) Italy, viale dei Cappuccini n.1, San Giovanni Rotondo, FG 71013, Italy. ${ }^{3}$ Unit of Biostatistics, DCPE, Consorzio Mario Negri Sud, Santa Maria Imbaro, CH, Italy. ${ }^{4}$ Euro-Mediterranean Institute of Science and Technology, Palermo, Italy. ${ }^{5}$ Department of Experimental Biomedicine and Clinical Neuroscience, University of Palermo, Palermo, Italy. ${ }^{6}$ Oncology Unit I.R.C.C.S. "Casa Sollievo della Sofferenza" Hospital San Giovanni Rotondo (FG) Italy, viale dei Cappuccini n.1, San Giovanni Rotondo, FG 71013, Italy. "Pathology Unit I.R.C.C.S. "Casa Sollievo della Sofferenza"
Hospital San Giovanni Rotondo (FG) Italy, viale dei Cappuccini n.1, San Giovanni Rotondo, FG 71013, Italy. ${ }^{8}$ University College London, Institute for Liver and Digestive Health, Division of Medicine, Royal Free Campus, London, UK. 'Division of Surgical Oncology "SS Annunziata" Hospital, Chieti, Italy.

Received: 6 May 2014 Accepted: 29 August 2014

Published online: 10 September 2014

\section{References}

1. Jemal A, Siegel R, Ward E, Hao Y, Xu J, Thun MJ: Cancer statistics, 2009. CA Cancer J Clin 2009, 59:225-249.

2. Landis SH, Murray T, Bolden S, Wingo PA: Cancer statistics, 1999. CA Cancer J Clin 1999, 49(1):8-31. 1

3. Warshaw AL, Fernández-del Castillo C: Pancreatic carcinoma. N Engl J Med 1992, 13;326(7):455-465.

4. Hariharan D, Saied A, Kocher HM: Analysis of mortality rates for pancreatic cancer across the world. HPB (Oxford) 2008, 10(1):58-62.

5. Abbruzzese JL: New applications of gemcitabine and future directions in the management of pancreatic cancer. Cancer 2002, 95(4 Suppl):941-945.

6. Rathos MJ, Joshi K, Khanwalkar H, Manohar SM, Joshi KS: Molecular evidence for increased antitumor activity of gemcitabine in combination with a cyclin-dependent kinase inhibitor, P276-00 in pancreatic cancers. J Transl Med 2012, 10:161.

7. Ballehaninna UK, Chamberlain RS: Biomarkers for pancreatic cancer: promising new markers and options beyond CA 19-9. Tumour Biol 2013, 34(6):3279-3292

8. Di Gangi I, Vrovsek U, Pazienza V, Mattivi F: Analytical metabolomics-based approaches to pancreatic cancer. Trends Anal Chem 2014, 55:94-116.

9. Zhang J, Visser F, King KM, Baldwin SA, Young JD, Cass CE: The role of nucleoside transporters in cancer chemotherapy with nucleoside drugs. Cancer Metastasis Rev 2007, 26(1):85-110.

10. Pérez-Torras S, García-Manteiga J, Mercadé E, Casado FJ, Carbó N, Pastor-Anglada M, Mazo A: Adenoviral-mediated overexpression of human equilibrative nucleoside transporter 1 (hENT1) enhances gemcitabine response in human pancreatic cancer. Biochem Pharmacol 2008, 76(3):322-329.

11. Wang H, Word BR, Lyn-Cook BD: Enhanced efficacy of gemcitabine by indole-3-carbinol in pancreatic cell lines: the role of human equilibrative nucleoside transporter 1. Anticancer Res 2011, 31(10):3171-3180.

12. Eto K, Kawakami H, Kuwatani M, Kudo T, Abe Y, Kawahata S, Takasawa A, Fukuoka M, Matsuno Y, Asaka M, Sakamoto N: Human equilibrative nucleoside transporter 1 and Notch3 can predict gemcitabine effects in patients with unresectable pancreatic cancer. Br J Cancer 2013, 108(7):1488-1494.

13. Santini D, Vincenzi B, Fratto ME, Perrone G, Lai R, Catalano V, Cass C, Ruffini PA, Spoto C, Muretto P, Rizzo S, Muda AO, Mackey JR, Russo A, Tonini G, Graziano F: Prognostic role of human equilibrative transporter 1 (hENT1) in patients with resected gastric cancer. J Cell Physiol 2010, 223(2):384-388.

14. Farrell JJ, Elsaleh H, Garcia M, Lai R, Ammar A, Regine WF, Abrams R, Benson AB, Macdonald J, Cass CE, Dicker AP, Mackey JR: Human equilibrative nucleoside transporter 1 levels predict response to gemcitabine in patients with pancreatic cancer. Gastroenterology 2009, 136(1):187-195.

15. Ohhashi S, Ohuchida K, Mizumoto K, Fujita H, Egami T, Yu J, Toma H, Sadatomi S, Nagai E, Tanaka M: Down-regulation of deoxycytidine kinase enhances acquired resistance to gemcitabine in pancreatic cancer. Anticancer Res 2008, 28(4B):2205-2212.

16. Noma B, Sasaki T, Fujimoto $Y$, Serikawa M, Kobayashi K, Inoue M, Itsuki H, Kamigaki M, Minami T, Chayama K: Expression of multidrug resistanceassociated protein 2 is involved in chemotherapy resistance in human pancreatic cancer. Int J Oncol 2008, 33(6):1187-1194.

17. Miller DW, Fontain M, Kolar C, Lawson T: The expression of multidrug resistance-associated protein (MRP) in pancreatic adenocarcinoma cell lines. Cancer Lett 1996, 107(2):301-306.

18. Su N, Kilberg MS: C/EBP homology protein (CHOP) interacts with activating transcription factor 4 (ATF4) and negatively regulates the stress-dependent induction of the asparagine synthetase gene. J Biol Chem 2008, 283(50):35106-35117.

19. Pandolfi A, Di Pietro N: High glucose, nitric oxide, and adenosine: a vicious circle in chronic hyperglycaemia? Cardiovasc Res 2010, 86(1):9-11.

20. Pazienza V, Tavano F, Benegiamo G, Vinciguerra M, Burbaci FP, Copetti M, di Mola FF, Andriulli A, di Sebastiano P: Correlations among PPARY, DNMT1, 
and DNMT3B Expression Levels and Pancreatic Cancer. PPAR Res 2012 2012:461784.

21. Rappa F, Greco A, Podrini C, Cappello F, Foti M, Bourgoin L, Peyrou M, Marino A, Scibetta N, Williams R, Mazzoccoli G, Federici M, Pazienza V, Vinciguerra M: Immunopositivity for histone macroH2A1 isoforms marks steatosis-associated hepatocellular carcinoma. PLoS One 2013, 8(1):e54458.

22. Ciampi A: Generalized Regression Tree. Comput Stat Data Anal 1991, 12(1):57-78.

23. Barbano R, Muscarella LA, Pasculli B, Valori VM, Fontana A, Coco M, la Torre A, Balsamo T, Poeta ML, Marangi GF, Maiello E, Castelvetere M, Pellegrini F, Murgo R, Fazio VM, Parrella P: Aberrant Keap1 methylation in breast cancer and association with clinicopathological features. Epigenetics 2013, 8(1):105-112.

24. Hagmann W, Jesnowski R, Löhr JM: Interdependence of Gemcitabine Treatment, Transporter Expression, and Resistance in Human Pancreatic Carcinoma Cells. Neoplasia 2010, 12:740-747.

25. Nishio R, Tsuchiya H, Yasui T, Matsuura S, Kanki K, Kurimasa A, Hisatome I, Shiota G: Disrupted plasma membrane localization of equilibrative nucleoside transporter 2 in the chemoresistance of human pancreatic cells to gemcitabine (dFdCyd). Cancer Sci 2011, 102:622-629.

26. Spratlin J, Sangha R, Glubrecht D, Dabbagh L, Young JD, Dumontet C, Cass C, Lai R, Mackey JR: The Absence of Human Equilibrative Nucleoside Transporter 1 Is Associated with Reduced Survival in Patients With Gemcitabine-Treated Pancreas Adenocarcinoma. Clin Cancer Res 2004, 10(20):6956-6961.

27. Nakagawa N, Murakami Y, Uemura K, Sudo T, Hashimoto Y, Kondo N, Sueda T: Combined analysis of intratumoral human equilibrative nucleoside transporter 1 (hENT1) and ribonucleotide reductase regulatory subunit M1 (RRM1) expression is a powerful predictor of survival in patients with pancreatic carcinoma treated with adjuvant gemcitabine-based chemotherapy after operative resection. Surgery 2013, 153(4):565-575.

28. Giovannetti E, Del Tacca M, Mey V, Funel N, Nannizzi S, Ricci S, Orlandini C, Boggi U, Campani D, Del Chiaro M, lannopollo M, Bevilacqua G, Mosca F, Danesi R: Transcription Analysis of Human Equilibrative Nucleoside Transporter-1 Predicts Survival in Pancreas Cancer Patients Treated with Gemcitabine. Cancer Res 2006, 66(7):3928-3935.

29. Benassai G1, Mastrorilli M, Quarto G, Cappiello A, Giani U, Forestieri P, Mazzeo F: Factors influencing survival after resection for ductal adenocarcinoma of the head of the pancreas. J Surg Oncol 2000, 73(4):212-218

30. Sohn TA1, Yeo CJ, Cameron JL, Koniaris L, Kaushal S, Abrams RA, Sauter PK, Coleman J, Hruban RH, Lillemoe KD: Resected adenocarcinoma of the pancreas-616 patients: results, outcomes, and prognostic indicators. J Gastrointest Surg 2000, 4(6):567-579.

31. Strasberg SM, Gao F, Sanford D, Linehan DC, Hawkins WG, Fields R, Carpenter DH, Brunt EM, Phillips C: Jaundice: an important, poorly recognized risk factor for diminished survival in patients with adenocarcinoma of the head of the pancreas. HPB (Oxford) 2014 16(2):150-156

32. Changchien CS, Yung CY, Tzen KY: Serum CEA and CA 19-9 value in patients with pancreatic cancer or pancreatitis. Changgeng Yi Xue Za Zhi 1991, 14(1):32-38.

33. Lundin J1, Roberts PJ, Kuusela P, Haglund C, Haglund: The prognostic value of preoperative serum levels of CA 19-9 and CEA in patients with pancreatic cancer. Br J Cancer 1994, 69(3):515-519.

34. Fukushima N, Hruban RH, Kato Y, Klimstra DS, Kloppel G, Shimizu N, Terris B: Ductal adenocarcinoma variants and mixed neoplasms of the pancreas. In WHO classification of tumors of the digestive system. Edited by Bosman FT, Carneiro F, Hruban RH, Theise ND. Lyon: IARC; 2008:292-299.
35. Greenhalf W, Ghaneh P, Neoptolemos JP, Palmer DH, Cox TF, Lamb RF, Garner E, Campbell F, Mackey JR, Costello E, Moore MJ, Valle JW, McDonald AC, Carter R, Tebbutt NC, Goldstein D, Shannon J, Dervenis C, Glimelius B, Deakin M, Charnley RM, Lacaine F, Scarfe AG, Middleton MR, Anthoney A, Halloran CM, Mayerle J, Oláh A, Jackson R, Rawcliffe CL, Scarpa A, Bassi C, Büchler MW, European Study Group for Pancreatic Cancer: Pancreatic cancer hENT1 expression and survival from gemcitabine in patients from the ESPAC-3 trial. J Nat/ Cancer Inst 2014, 106(1):djt347. doi:10.1093/jnci/djt347.

doi:10.1186/s12967-014-0248-4

Cite this article as: Tavano et al:: Modeling interactions between Human Equilibrative Nucleoside Transporter-1 and other factors involved in the response to gemcitabine treatment to predict clinical outcomes in pancreatic ductal adenocarcinoma patients. Journal of Translational Medicine 2014 12:248.

\section{Submit your next manuscript to BioMed Central and take full advantage of:}

- Convenient online submission

- Thorough peer review

- No space constraints or color figure charges

- Immediate publication on acceptance

- Inclusion in PubMed, CAS, Scopus and Google Scholar

- Research which is freely available for redistribution 